\title{
PRINCIPAL PIVOT TRANSFORMS OF RANGE SYMMETRIC MATRICES IN MINKOWSKI SPACE
}

\author{
AR. MEENAKSHI AND D. KRISHNASWAMY
}

\begin{abstract}
It is shown that the property of a matrix being range symmetric in Minkowski space $\mathfrak{m}$ is preserved under the principal pivot transformation.
\end{abstract}

\section{Introduction}

Throughout we shall deal with $C^{n \times n}$, the space of $n \times n$ complex matrices. Let $C^{n}$ be the space of complex $n$-tuples, we shall index the components of a complex vector in $C^{n}$ from 0 to $n-1$, that is $u=\left(u_{0}, u_{1}, u_{2}, \ldots, u_{n-1}\right)$. Let $G$ be the Minkowski metric tensor defined by $G u=\left(u_{0},-u_{1},-u_{2}, \ldots,-u_{n-1}\right)$. Clearly, the Minkowski metric matrix

$$
G=\left[\begin{array}{cc}
1 & 0 \\
0 & -I_{n-1}
\end{array}\right], \quad G=G^{*} \text { and } G^{2}=I_{n} .
$$

In [8], Minkowski inner product on $C^{n}$ is defined by $(u, v)=[u, G v]$, where [....] denotes the conventional Hilbert (unitary) space inner product. A space with Minkowski inner product is called a Minkowski space and denoted as $\mathfrak{m}$.

For $A \in C^{n \times n}, x, y \in C^{n}$ and by using (1.1), we get

$$
\begin{aligned}
(A x, y) & =[A x, G y] \\
& =\left[x, A^{*} G y\right] \\
& =\left[x, G\left(G A^{*} G\right) y\right] \\
& =\left[x, G A^{\sim} y\right] \\
& =\left(x, A^{\sim} y\right) \quad \text { where } A^{\sim}=G A * G .
\end{aligned}
$$

$A^{\sim}$ is called the Minkowski adjoint of $A$ in $\mathfrak{m}$ ( $A^{*}$ is usual Hermitian adjoint of $A$ ). Naturally, we call a matrix $A \in C^{n \times n} \mathfrak{m}$-symmetric in $\mathfrak{m}$ if $A=A^{\sim}$. For $A \in C^{n \times n}$, $\operatorname{rk}(A), N(A)$ and $R(A)$ are respectively the rank of $A$, the null space of $A$ and the range space of $A$. By a generalized inverse of $A$ we mean a solution of the equation $A \times A=A$ and is denoted as $A^{(1)} . A\{1\}$ is the set of all generalized inverses of $A$. Throughout $I$ refers to identity matrix of appropriate order unless otherwise specified.

Received January 12, 2005.

2000 Mathematics Subject Classification. 15A09, 15A57.

Key words and phrases. Minkowski space, principal pivot, range symmetric matrices. 
Definition 1.1.(p.7 [2]) For $A \in C^{m \times n}, A^{+}$is the Moore-Penrose inverse of $A$ if $A A^{+} A=A, A^{+} A A^{+}=A^{+}, A A^{+}$and $A^{+} A$ are hermitian.

Theorem 1.2.([4]) For $A, B, C \in C^{n \times n}$, the following are equivalent

(i) $C A^{(1)} B$ is invariant for every $A^{(1)} \in A\{1\}$.

(ii) $N(A) \subseteq N(C)$ and $N\left(A^{*}\right) \subseteq N\left(B^{*}\right)$.

(iii) $C=C A^{(1)} A$ and $B=A A^{(1)} B$ for every $A^{(1)} \in A\{1\}$.

Theorem 1.3.(Lemma $3.3[7])$ Let $A$ and $B$ be matrices in $\mathfrak{m}$. Then $N\left(A^{*}\right) \subseteq$ $N\left(B^{*}\right) \Leftrightarrow N\left(A^{\sim}\right) \subseteq N\left(B^{\sim}\right)$.

Theorem 1.4.(Lemma $2.3[7])$ For $A_{1}, A_{2} \in C^{n \times n}\left(A_{1} A_{2}\right)^{\sim}=A_{2}^{\sim} A_{1}^{\sim}$ and $\left(A_{1}^{\sim}\right)^{\sim}=$ $A_{1}$.

A matrix $A \in C^{n \times n}$ is said to be range symmetric is unitary space (or) equivalently $A$ is said to be $E P$ if $N(A)=N\left(A^{*}\right)$ [or $\left.A A^{+}=A^{+} A\right]$ [p.163(2)]. For further properties of EP matrices one may refer [1, 2, 4 and 9].

In [6], the concept of range symmetric matrix in $\mathfrak{m}$ is introduced and developed analogous to that of $E P$ matrices in unitary space. A matrix $A \in C^{n \times n}$ is said to be range symmetric in $m \Leftrightarrow N(A)=N\left(A^{\sim}\right)$. In the sequel, we shall make use of the following results.

Theorem 1.5. (Theorem $2.2[6])$ For $A \in C^{n \times n}$, the following are equivalent:

i) $A$ is range symmetric in $\mathfrak{m}$

ii) $G A$ is $E P$

iii) $A G$ is $E P$

iv) $N\left(A^{*}\right)=N(A G)$

v) $R(A)=R\left(A^{\sim}\right)$

vi) $A^{\sim}=H A=A K$ for some non-singular matrices $H$ and $K$.

vii) $R\left(A^{*}\right)=R(G A)$

Definition 1.6.(p.291 [3]) Let $M=\left[\begin{array}{cc}A & B \\ C & D\end{array}\right]$ be an $n \times n$ matrix. The schur complement of $A$ in $M$, denoted by $S$ is defined as $D-C A^{(1)} B$, where $A^{(1)}$ is a generalized inverse of $A$.

Theorem 1.7.(Theorem $1[4])$ Let $M=\left[\begin{array}{ll}A & B \\ C & D\end{array}\right]$ be an $n \times n$ matrix with $N(A) \subseteq$ $N(C)$ and $N(S) \subseteq N(B)$. Then $M$ is EP if and only if $A$ and $S$ the schur complement of $A$ in $M$ are $E P, N\left(A^{*}\right) \subseteq N\left(B^{*}\right)$ and $N\left(S^{*}\right) \subseteq N\left(C^{*}\right)$.

\section{Principal Pivot on a Matrix}

Let us consider a system of linear equations $M z=t$, where $M=\left[\begin{array}{ll}A & B \\ C & D\end{array}\right]$ satisfying 
$N(A) \subseteq N(C)$ and $N\left(A^{*}\right) \subseteq N\left(B^{*}\right)$. If $z$ and $t$ are partitioned conformally as $z=\left[\begin{array}{l}x \\ y\end{array}\right]$ and $t=\left[\begin{array}{l}u \\ v\end{array}\right]$ then the system becomes $A x+B y=u ; C x+D y=v$. Since $M$ satisfy $N(A) \subseteq N(C)$ and $N\left(A^{*}\right) \subseteq N\left(B^{*}\right)$ by using Theorem 1.2 and Theorem 1.3, we can express $x$ and $v$ in terms of $u$ and $y$ as $x=A^{+} u-A^{+} B y ; v=C A^{+} u-\left(D-C A^{+} B\right) y$. Thus $M=\left[\begin{array}{cc}A & B \\ C & D\end{array}\right]$ which satisfies $N(A) \subseteq N(C), N\left(A^{*}\right) \subseteq N\left(B^{*}\right)$ can be transformed into the matrix

$$
\hat{M}=\left[\begin{array}{cc}
A^{+} & -A^{+} B \\
C A^{+} & S
\end{array}\right]
$$

where $S=D-C A^{+} B$ is the schur complement of $A$ in $M . \hat{M}$ is called a principal pivot transform of $M$. The operation that transforms $M \rightarrow \hat{M}$ is called a principal pivot. If $A$ is non-singular it reduces to the principal pivot by pivoting the block $A$ [10]. Properties and applications of the principal pivot transforms are well recognized in mathematical programming [10 and 11].

Theorem 2.1. Let $M=\left[\begin{array}{ll}A & B \\ C & D\end{array}\right]$ be a matrix with $N(A) \subseteq N(C)$ and $N\left(A^{*}\right) \subseteq$ $N\left(B^{*}\right), S$ the schur complement of $A$ in $M$, then the following are equivalent:

i) $M$ is range symmetric in $\mathfrak{m}$.

ii) $A$ is range symmetric in $\mathfrak{m}$ and $S$ is $E P, N\left(A^{\sim}\right) \subseteq N\left(B^{\sim}\right)$ and $N\left(S^{\sim}\right) \subseteq N\left(C^{\sim}\right)$.

Proof. (i) $\Rightarrow$ (ii) Let us consider the matrices $P=\left[\begin{array}{cc}I & O \\ C A^{(1)} & I\end{array}\right] ; Q=\left[\begin{array}{cc}I & B S^{(1)} \\ O & I\end{array}\right]$ for $A^{(1)} \in A\{1\}$ and $S^{(1)} \in S\{1\}$, and $L=\left[\begin{array}{cc}A & O \\ O & S\end{array}\right] \cdot\left[\begin{array}{cc}A^{(1)} & O \\ O & S^{(1)}\end{array}\right]$ is one choice of $L^{(1)}$. $P, Q$ are non-singular. Since $N(A) \subseteq N(C), N(S) \subseteq N(B)$, by Theorem 1.2, we have $C=C A^{(1)} A$ and $B=B S^{(1)} S$. Thus $M$ can be factorized as

$$
\begin{aligned}
P Q L & =\left[\begin{array}{cc}
I & O \\
C A^{(1)} & I
\end{array}\right]\left[\begin{array}{ll}
I & B S^{(1)} \\
O & I
\end{array}\right]\left[\begin{array}{ll}
A & O \\
O & S
\end{array}\right] \\
& =\left[\begin{array}{ll}
A & B S^{(1)} S \\
C A^{(1)} A & C A^{(1)} B S^{(1)} S+S
\end{array}\right]=\left[\begin{array}{ll}
A & B S^{(1)} S \\
C A^{(1)} A & C A^{(1)} B+S
\end{array}\right]=M
\end{aligned}
$$

Since $M=P Q L, P$ and $Q$ are non-singular and $N(L) \subseteq N(M)$. Also $\operatorname{rk}(M)=$ $\operatorname{rk}(P Q L)=\operatorname{rk}(L)$ therefore $N(L)=N(M)$. Also $M$ is range symmetric in $\mathfrak{m}$, we have 
$N\left(M^{\sim}\right)=N(M)=N(L)$ hence by using Theorem 1.2, we get

$$
\begin{aligned}
M^{\sim} & =M^{\sim} L^{(1)} L \\
G M^{*} G & =G M^{*} G L^{(1)} L \\
M^{*} & =M^{*} G L^{(1)} L G \\
{\left[\begin{array}{ll}
A^{*} & C^{*} \\
B^{*} & D^{*}
\end{array}\right] } & =\left[\begin{array}{ll}
A^{*} & C^{*} \\
B^{*} & D^{*}
\end{array}\right]\left[\begin{array}{cc}
G_{1} & O \\
O & -I
\end{array}\right]\left[\begin{array}{cc}
A^{(1)} & O \\
O & S
\end{array}\right]\left[\begin{array}{cc}
A & O \\
O & S
\end{array}\right]\left[\begin{array}{cc}
G_{1} & O \\
O & -I
\end{array}\right] \\
{\left[\begin{array}{ll}
A^{*} & C^{*} \\
B^{*} & D^{*}
\end{array}\right] } & =\left[\begin{array}{ll}
A^{*} G_{1} A^{(1)} A G_{1} & C^{*} S^{(1)} S \\
B^{*} G_{1} A^{(1)} A G_{1} & D^{*} S^{(1)} S
\end{array}\right] .
\end{aligned}
$$

Equating the corresponding blocks, we get

$$
\begin{aligned}
A^{*} & =A^{*} G_{1} A^{(1)} A G_{1} \\
G_{1} A^{*} G_{1} & =G_{1} A^{*} G_{1} A^{(1)} A \\
A^{\sim} & =A^{\sim} A^{(1)} A
\end{aligned}
$$

[By using (1.2)]

By using Theorem 1.2 and Theorem 1.3, for $A^{\sim}=A^{\sim} A^{(1)} A$, we get $N(A) \subseteq N\left(A^{\sim}\right)$ also $\operatorname{rk}(A)=\operatorname{rk}\left(A^{\sim}\right)$ implies $N(A)=N\left(A^{\sim}\right)$. Thus $A$ is range symmetric in $\mathfrak{m}$.

$$
\begin{aligned}
B^{*} & =B^{*} G_{1} A^{(1)} A G_{1} \\
G_{1} B^{*} G_{1} & =G_{1} B^{*} G_{1} A^{(1)} A \\
B^{\sim} & =B^{\sim} A^{(1)} A .
\end{aligned}
$$

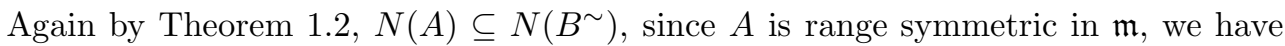
$N\left(A^{\sim}\right)=N(A) \subseteq N\left(B^{\sim}\right)$. Using $C^{*}=C^{*} S^{(1)} S$ and $D^{*}=D^{*} S^{(1)} S$ in $S=D-C A^{(1)} B$, we get

$$
\begin{aligned}
S^{*} S^{(1)} S & =\left(D-C A^{(1)} B\right)^{*} S^{(1)} S \\
& =D^{*} S^{(1)} S-\left(C A^{(1)} B\right)^{*} S^{(1)} S \\
& =D^{*} S^{(1)} S-B^{*}\left(A^{(1)}\right)^{*} C^{*} S^{(1)} S \\
& =D^{*}-B^{*}\left(A^{(1)}\right)^{*} C^{*} \\
& =\left(D-C A^{(1)} B\right)^{*} \\
S^{*} S^{(1)} S & =S^{*} .
\end{aligned}
$$

By applying Theorem 1.2, we get $N(S) \subseteq N\left(S^{*}\right)$. Since $\operatorname{rk}(S)=\operatorname{rk}\left(S^{*}\right), N(S)=$ $N\left(S^{*}\right)$ from this it follows that $S$ is $E P$. Again applying Theorem 1.2, for $C^{*}=C^{*} S^{(1)} S$ implies $N(S) \subseteq N\left(C^{*}\right)$ also $S$ is $E P$. We have $N\left(S^{*}\right)=N(S) \subseteq N\left(C^{*}\right)$. By using Theorem 1.3, we have $N\left(S^{\sim}\right) \subseteq N\left(C^{\sim}\right)$. Hence (ii) is proved.

(ii) $\Rightarrow$ (i) By hypothesis $A$ is range symmetric, $S$ is $E P, N(A) \subseteq N(C), N\left(A^{\sim}\right) \subseteq$ $N\left(B^{\sim}\right), N(S) \subseteq N(B)$ and $N\left(S^{\sim}\right) \subseteq N\left(C^{\sim}\right)$. Since $A$ is range symmetric in $\mathfrak{m}$ by 
Theorem 1.5 (ii), $G_{1} A$ is $E P$. Therefore

$$
\begin{aligned}
G_{1} A\left(G_{1} A\right)^{+} & =\left(G_{1} A\right)^{+} G_{1} A \\
G_{1} A A^{+} G_{1} & =A^{+} G_{1} G_{1} A \\
G_{1} A A^{+} G_{1} & =A^{+} A
\end{aligned}
$$

By using Theorem 1.2 and Theorem 1.3 we have $N(A) \subseteq N(C), N\left(A^{*}\right) \subseteq N\left(B^{*}\right)$, $N(S) \subseteq N(B)$ and $N\left(S^{*}\right) \subseteq N\left(C^{*}\right)$ hold. According to the assumption of Theorem 1 (v) [3], we have

$$
M^{+}=\left[\begin{array}{cc}
A^{+}+A^{+} B S^{+} C A^{+} & -A^{+} B S^{+} \\
-S^{+} C A^{+} & S^{+}
\end{array}\right]
$$

Under the condition $N\left(A^{\sim}\right) \subseteq N\left(B^{\sim}\right)$ and $N\left(S^{\sim}\right) \subseteq N\left(C^{\sim}\right)$, we have $A A^{+} B=B$, $C=S S^{+} C$

$$
\begin{aligned}
& \text { Now } M M^{+}=\left[\begin{array}{ll}
A & B \\
C & D
\end{array}\right]\left[\begin{array}{cc}
A^{+}+A^{+} B S^{+} C A^{+} & -A^{+} B S^{+} \\
-S^{+} C A^{+} & S^{+}
\end{array}\right] \\
& =\left[\begin{array}{ll}
A A^{+}+A A^{+} B S^{+} C A^{+}-B S^{+} C A^{+} & -A A^{+} B S^{+}+B S^{+} \\
C A^{+}+C A^{+} B S^{+} C A^{+}-D S^{+} C A^{+} & -C A^{+} B S^{+}+D S^{+}
\end{array}\right] \\
& =\left[\begin{array}{cc}
A A^{+}+A A^{+} B S^{+} C A^{+}-B S^{+} C A^{+} & -A A^{+} B S^{+}+B S^{+} \\
C A^{+}-\left(D-C A^{+} B\right) S^{+} C A^{+} & \left(D-C A^{+} B\right) S^{+}
\end{array}\right] \\
& M M^{+}=\left[\begin{array}{cc}
A A^{+} & O \\
O & S S^{+}
\end{array}\right]
\end{aligned}
$$

Similarly by using Theorem 1.2, $N(A) \subseteq N(C)$ and $N(S) \subseteq N(B)$, we have $C=$ $C A^{+} A ; B=B S^{+} S$,

$$
M^{+} M=\left[\begin{array}{cc}
A^{+} A & O \\
O & S^{+} S
\end{array}\right]
$$

We claim $G M$ is $E P$, for

$$
\begin{aligned}
& G M(G M)^{+}=G M M^{+} G \\
& =G\left[\begin{array}{cc}
A A^{+} & O \\
O & S S^{+}
\end{array}\right] G=\left[\begin{array}{cc}
G_{1} & O \\
O & -I
\end{array}\right]\left[\begin{array}{cc}
A A^{+} & O \\
O & S S^{+}
\end{array}\right]\left[\begin{array}{cc}
G_{1} & O \\
O & -I
\end{array}\right] \\
& =\left[\begin{array}{cc}
G_{1} A A^{+} G_{1} & O \\
O & S S^{+}
\end{array}\right] \\
& =\left[\begin{array}{cc}
A^{+} A & O \\
O & S^{+} S
\end{array}\right] \quad[\text { By using }(2.2) \text { and } S \text { is } E P] \\
& =M^{+} M=M^{+} G G M \quad \text { [By using (1.1)] }
\end{aligned}
$$

Hence $G M$ is $E P$ again by Theorem 1.5 (ii), $M$ is range symmetric in $\mathfrak{m}$.

Theorem 2.2. Let $M=\left[\begin{array}{ll}A & B \\ C & D\end{array}\right]$ be range symmetric in $\mathfrak{m}$ with $N(A) \subseteq N(C)$, $N\left(A^{\sim}\right) \subseteq N\left(B^{\sim}\right)$, then $\hat{M}$ the principal pivot transform of $M$ is range symmetric in $\mathfrak{m}$. 
Proof. Since $M$ is range symmetric in $\mathfrak{m}$ with $N(A) \subseteq N(C), N\left(A^{\sim}\right) \subseteq N\left(B^{\sim}\right)$ by applying Theorem 2.1, $A$ is range symmetric in $\mathfrak{m}$. Again by Theorem 1.5 (ii) both $G M$ and $G_{1} A$ are $E P$, where $G_{1}$ is Minkowski tensor of order as that of $A$. Thus

$$
G M=\left[\begin{array}{cc}
G_{1} & O \\
O & -I
\end{array}\right]\left[\begin{array}{ll}
A & B \\
C & D
\end{array}\right]=\left[\begin{array}{cc}
G_{1} A & G_{1} B \\
-C & -D
\end{array}\right] .
$$

Since $N(A) \subseteq N(C), N\left(G_{1} A\right)=N(A) \subseteq N(C)$ and $N\left(A^{\sim}\right) \subseteq N\left(B^{\sim}\right)$ implies $N\left(A^{\sim} G_{1}\right) \subseteq N\left(B^{\sim} G_{1}\right) \Rightarrow N\left(G_{1} A\right)^{\sim} \subseteq N\left(G_{1} B\right)^{\sim}$ [By Theorem 1.4]. Thus $N\left(G_{1} A\right) \subseteq$ $N(C)$ and $N\left(G_{1} A\right)^{\sim} \subseteq N\left(G_{1} B\right)^{\sim}$ hold for $G M$. Hence by using (2.1) GM can be transformed into its principal pivot

$$
\begin{aligned}
\hat{G M} & =\left[\begin{array}{cc}
A^{+} G_{1} & -A^{+} B \\
-C A^{+} G_{1} & -S
\end{array}\right] \\
\text { Now } \hat{M} G & =\left[\begin{array}{cc}
A^{+} & -A^{+} B \\
C A^{+} & S
\end{array}\right]\left[\begin{array}{cc}
G_{1} & O \\
O & -I
\end{array}\right]=\left[\begin{array}{cc}
A^{+} G_{1} & A^{+} B \\
C A^{+} G_{1} & -S
\end{array}\right] \\
\text { Consider } P & =\left[\begin{array}{cc}
I & O \\
O & -I
\end{array}\right] ; P^{\sim}=G P^{*} G=\left[\begin{array}{cc}
I & O \\
O & -I
\end{array}\right]=P^{*} \\
\text { Now } P \hat{M} G P^{*} & =\left[\begin{array}{cc}
I & O \\
O & -I
\end{array}\right]\left[\begin{array}{cc}
A^{+} G_{1} & A^{+} B \\
C A^{+} G_{1} & -S
\end{array}\right]\left[\begin{array}{cc}
I & O \\
O & -I
\end{array}\right] \\
& =\left[\begin{array}{cc}
A^{+} G_{1} & -A^{+} B \\
-C A^{+} G_{1} & -S
\end{array}\right]=G \hat{M}
\end{aligned}
$$

Since $G M$ is $E P$, by Theorem 1 [5], $\hat{G M}$ is $E P$. Hence $\hat{M} G=P^{*} G \hat{M} P$ is $E P$, again by Theorem 1.5 (iii) $\hat{M}$ is range symmetric in $\mathfrak{m}$.

Lemma 2.3. Let $M=\left[\begin{array}{cc}A & B \\ C & D\end{array}\right], G=\left[\begin{array}{cc}G_{1} & O \\ O & -I\end{array}\right]$ be partitioned in confirmity with that of $M . S$ and $S_{1}$ be the schur complements of $A$ and $D$ is $M$ respectively. Let $N(A) \subseteq N(C)$ and $N(D) \subseteq N(B)$, then the following are equivalent.

i) $M$ is range symmetric in $\mathfrak{m}$ with $N(S) \subseteq N(B), N\left(S_{1}\right) \subseteq N(C)$.

ii) $A, G_{1} D$ are range symmetric in $\mathfrak{m}, S, G_{1} S_{1}$ are $E P, N(A)=N\left(S_{1}\right) \subseteq N\left(B^{\sim}\right)$ and $N(D)=N(S) \subseteq N\left(C^{\sim}\right)$.

Proof. (i) $\Rightarrow$ (ii) Since $M$ is range symmetric in $\mathfrak{m}$ with $N(A) \subseteq N(C)$ and $N(S) \subseteq$ $N(B)$ by Theorem 2.1, $A$ is range symmetric in $\mathfrak{m}$ and $S$ is $E P, N\left(A^{\sim}\right) \subseteq N\left(B^{\sim}\right)$ and $N\left(S^{\sim}\right) \subseteq N\left(C^{\sim}\right)$. Since $A$ is range symmetric in $\mathfrak{m}, N(A)=N\left(A^{\sim}\right) \subseteq N\left(B^{\sim}\right)$ and $S$ is $E P$ implies $N(S)=N\left(S^{*}\right)$ by using Theorem 1.7. $N\left(S^{*}\right) \subseteq N\left(C^{*}\right)$ again by Theorem 1.3, $N\left(S^{\sim}\right) \subseteq N\left(C^{\sim}\right)$ and hence $N(S) \subseteq N\left(C^{\sim}\right)$. Since $M$ is range symmetric in $\mathfrak{m}$, by Theorem 1.5 (ii), $G M$ is $E P$. Then the principal rearrangement $P^{*} G M P=$ $\left[\begin{array}{cc}-D & -C \\ G_{1} B & G_{1} A\end{array}\right]$ is $E P$ with $N(D) \subseteq N(B)=N\left(G_{1} B\right)$ and $N\left(G_{1} S_{1}\right)=N\left(S_{1}\right) \subseteq N(C)$. Now the schur complement of $D$ in $P^{*} G M P$ is $G_{1} A-G_{1} B D^{+} C=G_{1}\left(A-B D^{+} C\right)=$ 
$G_{1} S_{1}$ and therefore by Theorem 1.7, $D$ and $S_{1}$ are $E P . N\left(D^{*}\right) \subseteq N\left(C^{*}\right), N\left(G_{1} S_{1}\right)^{*} \subseteq$ $N\left(G_{1} B\right)^{*}$. Since $D, G_{1} S_{1}$ are $E P$ by using Theorem 1.5 (ii), $G_{1} D$ and $S_{1}$ using Theorem 1.3 , it follows that $N\left(G_{1} D\right)^{\sim}=N\left(G_{1} D\right)=N(D) \subseteq N\left(C^{\sim}\right)$ and $N\left(S_{1}^{\sim}\right)=N\left(S_{1}\right) \subseteq$ $N\left(B^{\sim}\right)$. Since $A$ is range symmetric in $\mathfrak{m}$, by Theorem 1.5 (ii), $G_{1} A$ is $E P$ and hence $G_{1} A A^{+} G_{1}=A^{+} A$. Also $N(A) \subseteq N(C), N(S) \subseteq N(B), N\left(A^{\sim}\right) \subseteq N\left(B^{\sim}\right)$ and $N\left(S^{\sim}\right) \subseteq$ $N\left(C^{\sim}\right)$ hold, by applying Theorem 1 (v) [3], we have $M^{+}$of the form (2.3). By using Theorem 1.2 and Theorem 1.3, for the conditions $N\left(A^{\sim}\right) \subseteq N\left(B^{\sim}\right)$ and $N\left(S^{\sim}\right) \subseteq$ $N\left(C^{\sim}\right)$. We get $M M^{+}=\left[\begin{array}{cc}A A^{+} & O \\ O & S S^{+}\end{array}\right]$[By using (2.5)]. Since $N(A) \subseteq N(C), N(S) \subseteq$ $N(B), N\left(A^{\sim}\right) \subseteq N\left(B^{\sim}\right)$ and $N\left(S^{\sim}\right) \subseteq N\left(C^{\sim}\right)$ hold for $A$ as well as $D$ according to the assumptions of Theorem $1(\mathrm{v})[3], M^{+}$is also given by

$$
M^{+}=\left[\begin{array}{cc}
S_{1}^{+} & -A^{+} B S^{+} \\
-D^{+} C S_{1}^{+} & S^{+}
\end{array}\right] \text {. }
$$

Again by using Theorem 1.2 and Theorem 1.3, for $N\left(A^{\sim}\right) \subseteq N\left(B^{\sim}\right), N\left(D^{\sim}\right) \subseteq$ $N\left(C^{\sim}\right)$ we have $B=A A^{+} B$ and $C=D D^{+} C$, hence

$$
M M^{+}=\left[\begin{array}{cc}
A A^{+} & O \\
O & S S
\end{array}\right]=\left[\begin{array}{cc}
S_{1} S_{1}^{+} & O \\
O & S S^{+}
\end{array}\right]
$$

Since $M$ is range symmetric in $\mathfrak{m}$ by Theorem 1.5 (ii), $G M$ is $E P$, by using (2.7) $M M^{+}=G M^{+} M G$ implies $G_{1} A^{+} A G_{1}=G_{1} S_{1}^{+} S_{1} G_{1}$ and hence $N(A)=N\left(S_{1}\right)$. Similarly, using the formulae (2.4) and (2.8), we obtain two more expressions for $M^{+} M$ comparing the coresponding block yields $D^{+} D=S^{+} S$ which implies $N(D)=N(S)$. Thus (ii) holds.

(ii) $\Rightarrow$ (i) $N(S) \subseteq N(B)$ follows directly from $N(S)=N(D) \subseteq N(B)$. Similarly, $N\left(S_{1}\right) \subseteq N(C)$ follows from $N\left(S_{1}\right)=N(A) \subseteq N(C)$. Since $A, G_{1} D$ are range symmetric in $\mathfrak{m}$ and $S, G_{1} S_{1}$ are $E P$ satisfying $N(A) \subseteq N(C), N(S) \subseteq N(B), N(D) \subseteq N(B)$ and $N\left(S_{1}\right) \subseteq N(C)$. Hence by Theorem 2.1, $M$ is range symmetric in $\mathfrak{m}$. Thus (i) holds.

Theorem 2.4. Let $M=\left[\begin{array}{ll}A & B \\ C & D\end{array}\right], S$ and $S_{1}$ be the schur complements of $A$ and $D$ in $M$ respectively, If $M$ is range symmetric in $\mathfrak{m}$ with $N(A) \subseteq N(C), N(D) \subseteq N(B)$, $N(S) \subseteq N(B)$ and $N\left(S_{1}\right) \subseteq N(C)$. The following hold:

i) Principal submatrices $A$ is range symmetric in $\mathfrak{m}$ and $D$ is EP.

ii) The schur complements $S$ and $G_{1} S_{1}$ are EP.

iii) The principal pivot transform $\hat{M}$ of $M$ by pivoting the block $A$ is range symmetric in $\mathfrak{m}$ and $\operatorname{rk}(\hat{M})=r$.

Proof. (i) and (ii) are consequence of Lemma 2.3.

(iii) By Lemma 2.3, $M$ satisfies $N(A) \subseteq N(C)$ and $N(S) \subseteq N(B)$ hence by pivoting the block $A$, the principal pivot transform $\hat{M}$ of $M$ is $\hat{M}=\left[\begin{array}{cc}A^{+} & -A^{+} B \\ C A^{+} & S\end{array}\right]$. In $\hat{M}$, 
$N\left(A^{+}\right) \subseteq N\left(C A^{+}\right)$and $N\left(A^{+}\right)^{*} \subseteq N\left(A^{+} B\right)^{*}$. Futher the schur complement of $A^{+}$in $\hat{M}$ is $\hat{S}=S+C A^{+}\left(A^{+}\right)^{+} A^{+} B=S+C A^{+} B=D$. By assumption $N(\hat{S})=N(D) \subseteq N(B)$. By using Lemma 2.3, $A$ and $G_{1} D$ are range symmetric in $\mathfrak{m}$. Again by Theorem 1.5 (ii), $G_{1} A$ and $D$ are $E P . N\left(D^{*}\right)=N(\hat{S})^{*} \subseteq N\left(C^{*}\right)$, by using Theorem 1.3, we get $N\left(\hat{S}^{\sim}\right) \subseteq N\left(C^{\sim}\right)$. Now applying Theorem 2.2 , we have $\hat{M}$ is range symmetric in $\mathfrak{m}$. Finally, we prove $\operatorname{rk}(\hat{M})=\operatorname{rk}(M)=r$. The proof runs as follows:

$$
\begin{aligned}
\operatorname{rk}(\hat{M}) & =\operatorname{rk}\left(A^{+}\right)+\operatorname{rk}(\hat{S}) \\
& =\operatorname{rk}(A)+\operatorname{rk}(D) \\
& =\operatorname{rk}(A)+\operatorname{rk}(S) \\
& =\operatorname{rk}(M)=r .
\end{aligned}
$$

$[$ By using $N(D)=N(S)]$

Remark 2.5. In the special case, when $M$ is non-singular with $A$ and $D$ nonsingular then the conditions $N(A) \subseteq N(C)$ and $N(D) \subseteq N(B)$ automatically hold and by Theorem 1 in [3], $S$ and $S_{1}$ are non-singular further $\operatorname{rk}(\hat{M})=\operatorname{rk}(A)+\operatorname{rk}(D)$. Hence it follows that the principal pivot transform $\hat{M}$ of $M$ if non-singular. However, we note that the non-singularity of $\hat{M}$ need not imply that $M$ is non-singular. This is illustrated in the following example.

$$
\begin{aligned}
\text { Example 2.6. Let } M & =\left[\begin{array}{llll}
1 & 0 & 0 & 0 \\
0 & 1 & 1 & 0 \\
0 & 1 & 2 & 1 \\
0 & 0 & 1 & 1
\end{array}\right] \text { be range symmetric in } \mathfrak{m} . \\
\text { For } M^{\sim}=G M^{*} G & =\left[\begin{array}{cccc}
1 & 0 & 0 & 0 \\
0 & -1 & 0 & 0 \\
0 & 0 & -1 & 0 \\
0 & 0 & 0 & -1
\end{array}\right]\left[\begin{array}{llll}
1 & 0 & 0 & 0 \\
0 & 1 & 1 & 0 \\
0 & 1 & 2 & 1 \\
0 & 0 & 1 & 1
\end{array}\right]\left[\begin{array}{cccc}
1 & 0 & 0 & 0 \\
0 & -1 & 0 & 0 \\
0 & 0 & -1 & 0 \\
0 & 0 & 0 & -1
\end{array}\right] \\
& =\left[\begin{array}{llll}
1 & 0 & 0 & 0 \\
0 & 1 & 1 & 0 \\
0 & 1 & 2 & 1 \\
0 & 0 & 1 & 1
\end{array}\right]=M
\end{aligned}
$$

Thus $N(M)=N\left(M^{\sim}\right)$ implies $M$ is range symmetric in $\mathfrak{m}$.

$$
\text { Let } B=C^{*}=\left[\begin{array}{ll}
0 & 0 \\
1 & 0
\end{array}\right] ; D=\left[\begin{array}{ll}
2 & 1 \\
1 & 1
\end{array}\right] \text { and } A=\left[\begin{array}{ll}
1 & 0 \\
0 & 1
\end{array}\right] \text {. Here } A \text { and } D \text { are non-singular }
$$

$$
\text { and } \begin{aligned}
S & =\left[\begin{array}{ll}
2 & 1 \\
1 & 1
\end{array}\right]-\left[\begin{array}{ll}
0 & 1 \\
0 & 0
\end{array}\right]\left[\begin{array}{ll}
1 & 0 \\
0 & 1
\end{array}\right]\left[\begin{array}{ll}
0 & 0 \\
1 & 0
\end{array}\right] \\
S & =\left[\begin{array}{ll}
2 & 1 \\
1 & 1
\end{array}\right]-\left[\begin{array}{ll}
1 & 0 \\
0 & 0
\end{array}\right]=\left[\begin{array}{ll}
1 & 1 \\
1 & 1
\end{array}\right]
\end{aligned}
$$


By Theorem 2.4, $S$ is $E P$ and $\operatorname{rk}(S)=1$ and hence $S$ is $E P_{1}$. Therefore $\operatorname{rk}(M)=$

$\operatorname{rk}(A)+\operatorname{rk}(S)=2+1=3$. By using $(2.1), \hat{M}=\left[\begin{array}{cccc}1 & 0 & 0 & 0 \\ 0 & 1 & -1 & 0 \\ 0 & 1 & 1 & 1 \\ 0 & 0 & 1 & 1\end{array}\right]$ is non-singular and by

using Theorem 2.4 $\operatorname{rk}(\hat{M})=\operatorname{rk}(A)+\operatorname{rk}(D)=2+2=4$.

\section{Acknowledgements}

The first author is thankful to the All India Council for Technical Education, New Delhi for the financial support to carryout the work.

\section{References}

[1] T. S. Baskett and I. J. Katz, Theorems on product of EPr matrices, Lin. Alg. Appln. 2(1969), 87-103.

[2] A. Ben Israel and T. N. E. Greville, Generalized Inverses, Theory and Application, Wiley and Sons, New York, 1974.

[3] D. Carlson, Emilie Haynsworth and Thomas Markham, A generalization of the schur complement by the Moore-Penrose inverse, SIAM. J. Appl. Math. 26(1974), 169-175.

[4] AR. Meenakshi, On schur complement in an EP matrix, Periodica Mathematica Hungarica 16(1985), 193-200.

[5] AR. Meenakshi, Principal pivot transform of an EP matrix, C. R. Math. Rep. Acad. Sci. 2(1986), 121-126.

[6] AR. Meenakshi, Range symmetric matrices in Minkowski space, Bull. Malaysian Math. Sci. Soc. 1(2000), 45-52.

[7] AR. Meenakshi and D. Krishnaswamy, On sums of range symmetric matrices in Minkowski space, Bull. Malaysian Math. Sci. Soc. Second Series 25(2002), 137-148.

[8] Michael Renardy, Singular value decomposition in Minkowski space, Lin. Alg. Appln. 236 (1996), 53-58.

[9] M. H. Pearl, On Normal and EPr matrices, Michigan Math. J. 6(1959), 1-5.

[10] A. W. Tucker, Combinatorial Analysis (Bellman and Hall Eds), American Math. Soc. Providence RI(1960), 129-140.

[11] A. W. Tucker, Principal pivot transforms of square matrices, SIAM. Rev. 5(1963), 305.

AICTE - Emeritus Professor of Mathematics, Faculty of Engineering and Technology, Annamalai University, Annamalai Nagar - 608 002. Tamil Nadu, SouthIndia.

E-mail: arm_meenakshi@yahoo.co.in

Reader in Mathematics, Directorate of Distance Education, Annamalai University, Annamalai Nagar - 608 002. Tamil Nadu, SouthIndia.

E-mail: Krishna_swamy2004@yahoo.co.in 